\title{
Do Giant Adrenal Myelolipomas Behave Differently? A Clinicopathological Comparative Study
}

Pooja Ramakant, Rajiv Mukha, Anish Jacob Cherian, Dukhabandhu Naik, Thomas V Paul, Deepak T Abraham, MJ Paul

\begin{abstract}
Adrenal myelolipomas (AM) may present with abdominal distension, pain, rupture, hemorrhage or other complications. Indications for surgery are still debatable. It is possible that larger size may be a risk factor for symptoms or complications in AM. To compare the clinical and pathological characteristics between giant $A M(>10 \mathrm{~cm})$ and $A M<10 \mathrm{~cm}$. Retrospective data analysis was done on 26 of 32 patients with myelolipoma who underwent surgery between January, 2005 and July, 2012. Patients were divided in two groups: Group $1(n=14)$ with giant $A M>10 \mathrm{~cm}$ and group $2(n=12) A M<10 \mathrm{~cm}$. Results were analyzed using independent sample t-test (SPSS v.17). Twenty-six myelolipomas managed surgically were identified from 338 adrenal tumors. None were functioning on biochemical evaluation. Comparing group 1 vs 2, the differences noted were: Commonest age group at presentation was 4th decade vs 5th decade, clinical presentation with abdominal pain was 11 vs 7 ( $p=0.05)$, surgical approach was open in $11 \mathrm{vs} 6$, and laparoscopic in 3 vs $6(p=0.03)$, mean tumor size was 15.57 vs $6.67 \mathrm{~cm}$, mean tumor weight $794 \mathrm{vs} 93 \mathrm{gm}$, internal hemorrhage on histopathology in 14 vs $10(p=0.05)$. Giant AM have a significantly higher rate of symptomatic presentation, pathological features of hemorrhage and necrosis and requirement for open surgery as compared to myelolipomas $<10 \mathrm{~cm}$ in our series.
\end{abstract}

Keywords: Giant adrenal myelolipoma, Hemorrhage, Laparoscopic adrenalectomy.

How to cite this article: Ramakant $P$, Mukha R, Cherian AJ, Naik D, Paul TV, Abraham DT, Paul MJ. Do Giant Adrenal Myelolipomas Behave Differently? A Clinicopathological Comparative Study. World J Endoc Surg 2013;5(1):12-15.

Source of support: Nil

Conflict of interest: None

\section{INTRODUCTION}

Adrenal myelolipomas (AM) are rare, classically nonfunctioning, benign adrenal tumors. They constitute 3 to $5 \%$ of all primary adrenal tumors. ${ }^{1,2}$ In the period before availability of abdominal imaging, such tumors were detected mostly on autopsy. Currently, myelolipomas are more commonly detected incidentally on abdominal imaging and constitute up to 10 to $15 \%$ of all adrenal incidentalomas. ${ }^{2,3}$

Patients may present with a dragging type of abdominal pain or distension especially in larger sized tumors. ${ }^{4}$ There are few case reports documenting presentations with virilization, hypertension, hyperaldosteronism and hypercortisolism indicating hormone secretion. ${ }^{5,6}$ Malignancy has not been reported in such lesions even when they attain large sizes. Hence, there are differing opinions regarding the indications and timing of surgery even in large tumors. Some centres prefer observation with annual imaging while others prefer surgery. ${ }^{7,8}$ As these tumors have a very indolent behavior in terms of increase in size more patients are now being offered observation. However, the data are very scarce on the natural history of giant AM and their behavior patterns in terms of patient safety especially in the conservatively managed group. The natural history of myelolipomas has not been clearly understood in the available literature to safely recommend observation for these lesions. It is conceivable that larger size may have some risk of complications like rupture or hemorrhage. Decisions and timing of surgery in AM are also not very clear due to limited data on such rare tumors. We performed a retrospective study to analyze the differences in the behavior patterns between the giant AM $(>10 \mathrm{~cm})$ and myelolipomas which were less than $10 \mathrm{~cm}$.

\section{MATERIALS AND METHODS}

A total of 32 cases of adrenal myelolipoma were identified on retrospective review of 338 adrenal case records of endocrine surgery, endocrinology and urology services during the period from January, 2005 to July, 2012. Complete electronic medical records on clinical presentation, operative treatment and histopathology were available on 26 patients. Six remaining patients were managed conservatively for different reasons and were not included in the study. The management decisions were made in a multidisciplinary meeting between medical and surgical teams. All patients underwent biochemical assessment in the form of serum or urine free cortisol, 24-hour urinary vanillylmandelic acid or metanephrine/normetanephrine and serum electrolytes. The lesions were imaged using contrastenhanced computerized tomography (CECT) in 23 patients and magnetic resonance imaging (MRI) done in three patients (one detected during pregnancy, one with coexistent sigmoid colon carcinoma and one incidental myelolipoma in a patient who underwent MRI to assess injury from a fall).

Patients who underwent surgery were divided in two groups: Group $1(\mathrm{n}=14)$ with tumor size $>10 \mathrm{~cm}$ and group $2(n=12)$ with patients having tumor size less than $10 \mathrm{~cm}$. 
Data were analyzed with the help of independent sample t-test (SPSS v 17).

\section{RESULTS}

Adrenal myelolipoma $(n=32)$ comprised $9.4 \%$ of primary adrenal tumors $(\mathrm{n}=338)$ managed in the study period. The 26 patients analyzed in two groups showed the following characteristics: Table 1 shows the comparison of patient profile, clinical presentations and surgical approaches in the two groups. In group 1, $78.6 \%$ patients were symptomatic in the form of abdominal pain or distension compared to group 2 (tumors $<10 \mathrm{~cm}$ ) with $58 \%$ patients $(\mathrm{p}=0.05)$. One patient in group 1 had an acute presentation with severe pain and urinary retention requiring hospitalization; the tumor was excised 2 months later, showing evidence of internal hemorrhage on imaging and pathology. Patients with giant myelolipomas presented on average a decade earlier (4th decade as compared to 5th decade) ( $p=0.03)$. There was no gender preponderance in either of the two groups. All of these myelolipomas were nonfunctional on biochemical assessment.

CECT abdomen was suggestive of myelolipoma in all patients; the typical features were well circumscribed, nonhomogenous, hypodense adrenal mass with fat and no or minimal enhancement after contrast medium. In only three patients a differential diagnosis of soft tissue sarcoma was considered due to large size, hemorrhage and heterogeneous density. Interestingly, one tumor of $2.3 \times$ 1.8 was detected by a histological surprise following radical nephrectomy for renal cell carcinoma; the tumor was not seen on preoperative CT or at surgery.

Associated diagnoses included obesity $(n=7)$, primary hypertension $(\mathrm{n}=8)$, diabetes mellitus $(\mathrm{n}=5)$, pregnancy $(n=1)$, cholelithiasis $(n=3)$, renal cell carcinoma $(n=1)$, sigmoid colon carcinoma $(n=1)$, pancreatic cancer $(n=1)$ and incisional hernia $(\mathrm{n}=1)$.

Table 1 also shows the different surgical approaches used in both the groups. With larger tumors the open approach is more common as expected. An en bloc resection was performed in one patient in each group respectively. In Group 1, one patient underwent en bloc resection including spleen, left kidney and tail of pancreas suspecting malignant infiltration. In group 2, one patient required additional nephrectomy due to inadvertent ligation of renal vessels. Laparoscopic excision was more frequent with smaller group 2 tumors; however, it was attempted in two patients with giant tumors, one patient needing conversion due to excessive bleeding.

Postoperative morbidity included wound dehiscence and urinary tract infection (UTI) in group 2; intra-abdominal collection, UTI, inadvertent pleural tear and abdominal distension in group 1.
Figure 1 shows internal hemorrhage in a histopathological specimen of a giant adrenal myelolipoma. Cut section of the gross specimen showed variegated appearance with yellow areas representing fat admixed with red brown areas representing the myeloid elements. In final histopathology, none had malignancy in either of the two groups. Histopathology was compared in both the groups (Table 2) and we found that there was more internal hemorrhage and necrosis in group 1 , pain was associated more commonly with internal hemorrhage (Table 3). None of our patients had rupture of tumor or external hemorrhage as presenting complaints.

\section{DISCUSSION}

To have a better understanding of the pattern of growth or functionality of AM and appropriate treatment recommendations, we need to prospectively study the natural history of these tumors when patients are kept on observation and assessed clinically and imaged annually. Han et al have studied the natural history of AM and out of 21 tumors, only four patients were operated on initially and three patients managed conservatively needed later surgery due to discomfort caused by tumor growth with mean follow-up of 3.2 years. ${ }^{8}$ In their study period, none had any adverse event and all tumors remained hormonally inactive. In our series during this study period, six patients with AM were kept on conservative follow-up but due to short-term follow-up only, we were not able to study and comment on the natural history.

The etiopathogenesis of myelolipomas is unclear; theories of origin include autonomous proliferation of bone marrow cells during embryogenesis, degeneration of adrenal cortical epithelial cells or bone marrow emboli. ${ }^{9}$ Myelolipomas are usually located in adrenal gland but occasionally they may be located at extra-adrenal sites like

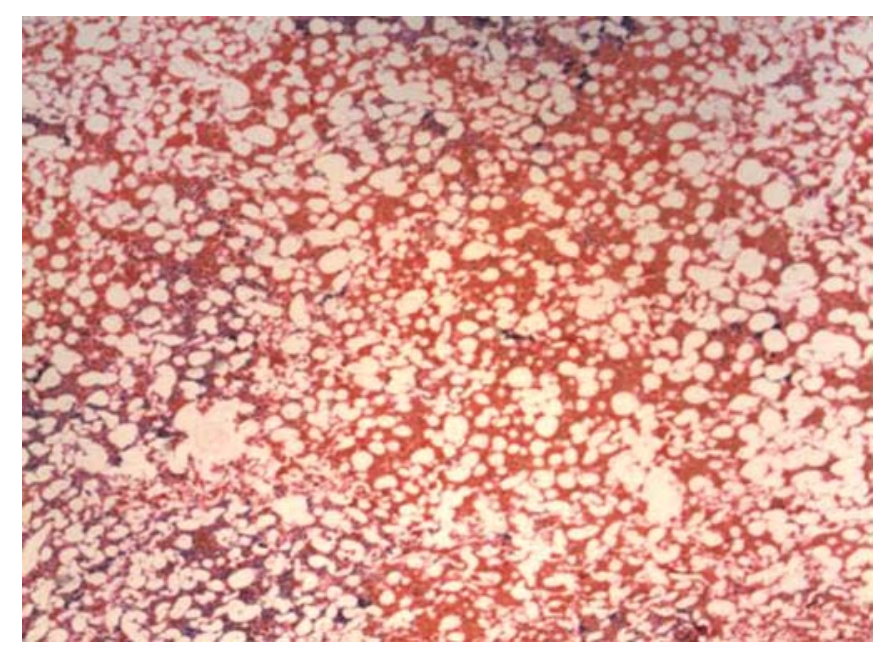

Fig. 1: Histopathological specimen of giant adrenal myelolipoma with internal hemorrhage 


\begin{tabular}{|c|c|c|c|}
\hline Patient profile & Group 1 (tumor $\geq 10 \mathrm{~cm})(n=14)$ & Group 2 (tumor size $<10 \mathrm{~cm})(n=12)$ & $p$-value \\
\hline $\begin{array}{l}\text { Mean age in years, range } \\
\text { Gender }\end{array}$ & 41.57 years $(21-55)$ & 51.17 years $(28-70)$ & 0.03 \\
\hline - Male & 6 & 7 & 0.8 \\
\hline - Female & 6 & 7 & \\
\hline Abdominal pain & $11(78.6 \%)$ & $7(58 \%)$ & 0.05 \\
\hline Open adrenalectomy & 12 (one converted) & & \\
\hline Laparoscopic adrenalectomy & 2 & 6 & 0.03 \\
\hline
\end{tabular}

\begin{tabular}{llll}
\multicolumn{5}{c}{ Table 2: Comparison of tumor characteristics in both the groups } \\
\hline Tumor characteristics & Group 1 & Group 2 & p-value \\
\hline Mean tumor size in cm (range) & $15.57(11-27)$ & $6.67(2-9)$ & $93.11(18-160)$ \\
Mean tumor weight in grams (range) & $794.29(100-4500)$ & 10 & 0.05 \\
Internal hemorrhage & 14 & 4 & 0.05 \\
Necrosis & 7 & 2 & 0.24 \\
Calcification & 5 & & \\
\hline
\end{tabular}

\begin{tabular}{lccc}
\multicolumn{2}{c}{ Table 3: Correlating pain and internal hemorrhage } \\
\hline Groups & Hemorrhage & \multicolumn{3}{c}{ Pain } \\
\cline { 3 - 4 } & & No & Yes \\
\hline $1(n=14)$ & No & 3 & 0 \\
Tumor $\geq 10 \mathrm{~cm}$ & Yes & 5 & 4 \\
$2(n=12)$ & No & 0 & 0 \\
Tumor $<10 \mathrm{~cm}$ & Yes & 12 & 2 \\
\hline
\end{tabular}

retroperitoneal presacral region, preirenal, renal hilum, spleen, lung and mediastinum. ${ }^{10}$ However, we have not encountered extra-adrenal myelolipomas in this study.

None of the tumors in this study period had clinical or biochemical evidence of hormonal secretion, however, there are case reports of myelolipomas associated with endocrine dysfunction like Cushing's syndrome, Addison's disease, congenital adrenal hyperplasia (CAH), hyperaldosteronism or pheochromocytoma. ${ }^{11}$ In $\mathrm{CAH}$, increased adrenocorticotrophic hormone levels may act like growth factors promoting development and growth of AM. However, the relationship of endocrine dysfunction and development of AM is not well understood.

Clinical presentation is usually with either dragging dull type abdominal pain due to large tumor mass or acute severe pain due to hemorrhage or rupture. Abdominal pain may be due to compression of the surrounding structures, stretching of capsule of the tumor, hemorrhage, necrosis or rupture. ${ }^{12}$ There are few case reports mentioning symptomatic external rupture with retroperitoneal hemorrhage. ${ }^{13}$ In our study, none had external hemorrhage but many patients in both the groups had internal hemorrhage which was evident on imaging and histopathology. Myeloid cells in AM may be responsible for hemorrhage.

In our series, CECT abdomen proved to be adequate for the diagnosis of myelolipomas. Rarely, a differential diagnosis has to be considered including liposarcoma, renal angiomyolipoma, metastasis, adrenal malignancy or adenoma due to large size, heterogeneous density, hemorrhage and few specks of calcification on imaging. ${ }^{14,15}$ Myelolipomas are usually well-circumscribed and noninfiltrative unlike liposarcomas. Unlike other functional endocrine tumors which are diagnosed biochemically, myelolipomas are well-diagnosed on imaging and hence, fine needle aspiration cytology has a very limited role in diagnosing such tumors. Whenever, there is suspicion of malignancy or other pathology on imaging then there is role of image guided needle biopsy. ${ }^{16}$ Where diagnostic dilemma persists, surgical excision for definitive histopathology may be required.

In asymptomatic patients, conservative treatment is recommended with follow-up by imaging. In symptomatic patients, those with large tumors or where there is suspicion of malignancy, surgery is recommended. ${ }^{17}$ When there is no suspicion of malignancy, a laparoscopic approach is recommended even in large tumors. ${ }^{17,18}$ Recently Shen et al have done retroperitoneal liposuction of large AM (all more than $8 \mathrm{~cm}$ ) successfully in eight patients. ${ }^{19}$

Limitations of this study were its retrospective nature and lack of adequate follow-up in patients managed conservatively to elucidate the natural history.

\section{CONCLUSION}

We recommend observation for asymptomatic patients and offer surgery only for those who are symptomatic or whose radiologic assessment is nondiagnostic. Tumor size in myelolipomas unlike other adrenal tumors is not a definite indication for surgery. However, the patients need to be counselled about the small risk of continued growth, rupture and internal/external hemorrhage when offering 
observation. Giant myelolipomas are more symptomatic and may need more open resections compared to myelolipomas with size $<10 \mathrm{~cm}$. Hence, treatment strategy may be individualized to patient needs and characteristics.

\section{REFERENCES}

1. Wani NA, Kosar T, Rawa IA, Qayum A. Giant adrenal myelolipoma: Incidentaloma with a rare incidental association. Urol Ann 2010 Sep;2(3):130-33.

2. Daneshmand S, Quek ML. Adrenal myelolipoma: Diagnosis and management. Urol J 2006 Spring;3(2):71-74.

3. Bhargav PR, Mishra A, Agarwal G, et al. Adrenal incidentalomas: Experience in a developing country. World J Surg 2008 Aug;32(8):1802-08.

4. Mondal SK, Sengupta S, Biswas PK, Sinha MG. Adrenal myelolipoma with abdominal pain: A rare presentation. Indian J Endocrinol Metab 2011 Jan;15(1):57-59.

5. German-Mena E, Zibari GB, Levine SN. Adrenal myelolipomas in patients with congenital adrenal hyperplasia: Review of the literature and a case report. Endocr Pract 2011 May-Jun;17(3): 441-47.

6. Ahsan T, Kanwal S, Banu Z, Jabeen R. Virilization with adrenal myelolipoma, adrenal hyperplasia, and fibroadenoma of breast. J Coll Physicians Surg Pak 2010 Dec;20(12):819-21.

7. Lam KY, Lo CY. Adrenal lipomatous tumours: A 30-year clinicopathological experience at a single institution. J Clin Pathol 2001 Sep;54(9):707-12.

8. Han M, Burnett AL, Fishman EK, Marshall FF. The natural history and treatment of adrenal myelolipoma. J Urol 1997 Apr;157(4):1213-16.

9. Mukherjee S, Pericleous S, Hutchins RR, Freedman PS. Asymptomatic giant adrenal myelolipoma. Urol J 2010 Winter; 7(1):66-68.

10. Beiko D, Roldan H, Sengupta SK, George RL. Laparoscopic excision of a large extra-adrenal perirenal myelolipoma. Can Urol Assoc J 2010 Apr;4(2):E39-41.

11. Mermejo LM, Elias Junior J, Saggioro FP, et al. Giant adrenal myelolipoma associated with 21-hydroxylase deficiency: Unusual association mimicking an androgen-secreting adrenocortical carcinoma. Arq Bras Endocrinol Metabol 2010 Jun;54(4):419-24.

12. Chakrabarti I, Ghosh N, Das V. Giant adrenal myelolipoma with hemorrhage masquerading as retroperitoneal sarcoma. J Midlife Health 2012 Jan;3(1):42-44.

13. Catalano O. Retroperitoneal hemorrhage due to a ruptured adrenal myelolipoma. A case report. Acta Radiol 1996 Sep; 37(5):688-90.

14. Fernandes GC, Gupta RK, Kandalkar BM. Giant adrenal myelolipoma. Indian J Pathol Microbiol 2010 Apr-Jun;53(2): 325-26.
15. Kiriakopoulos A, Tsakayannis D, Linos D. Surgical management of adrenal myelolipoma: A series of 10 patients and review of the literature. Minerva Chir 2006 Jun;61(3):241-46.

16. Settakorn J, Sirivanichai C, Rangdaeng S, Chaiwun B. Fineneedle aspiration cytology of adrenal myelolipoma: Case report and review of the literature. Diagn Cytopathol 1999 Dec;21(6): 409-12.

17. Ghatak S, Mridha AR. Laparoscopic resection of a large adrenal myelolipoma: A case report. Cases J 2009 Dec 11;2:9313.

18. Al Harthi B, Riaz MM, Al Khalaf AH, et al. Adrenal myelolipoma a rare benign tumour managed laparoscopically: Report of two cases. J Minim Access Surg 2009 Oct;5(4):118-20.

19. Shen X, Qiu Y, Zheng Y, Zhang S. retroperitoneal laparoscopic liposuction for large adrenal myelolipomas: A report of nine cases. J Laparoendosc Adv Surg Tech A 2012 Jul;22(6): $578-80$.

\section{ABOUT THE AUTHORS}

\section{Pooja Ramakant}

Associate Professor, Department of Endocrine Surgery, Christian Medical College, Vellore, Tamil Nadu, India

\section{Rajiv Mukha}

Associate Professor, Department of Urology, Christian Medical College, Vellore, Tamil Nadu, India

\section{Anish Jacob Cherian}

Registrar, Department of Endocrine Surgery, Christian Medical College, Vellore, Tamil Nadu, India

\section{Dukhabandhu Naik}

Assistant Professor, Department of Endocrinology, Christian Medical College, Vellore, Tamil Nadu, India

\section{Thomas V Paul}

Professor, Department of Endocrinology, Christian Medical College Vellore, Tamil Nadu, India

\section{Deepak T Abraham}

Professor, Department of Endocrine Surgery, Christian Medical College, Vellore, Tamil Nadu, India

\section{MJ Paul (Corresponding Author)}

Professor and Head, Department of Endocrine Surgery, Christian Medical College, Vellore, Tamil Nadu, India, e-mail:mjpaul@cmcvellore.ac.in 